\title{
Telessaúde como eixo organizacional dos sistemas universais de saúde do século XXI
}

\section{Telehealth as core of $21^{\text {st }}$ century universal health care systems}

\section{Telesalud como eje organizacional de los sistemas de salud universales del siglo XXI}

Erno Harzheim ${ }^{1}\left(\mathbb{D}\right.$, Patrícia Sampaio Chueiri ${ }^{\circledR}$, Roberto Nunes Umpierre ${ }^{1} \mathbb{D}$, Marcelo Rodrigues Gonçalves ${ }^{2} \mathbb{D}$, Ana Célia da Silva Siqueira ${ }^{3} \mathbb{D}$, Otávio Pereira D’Avila ${ }^{\circledR}$, Cynthia Goulart Molina Bastos ${ }^{5} \mathbb{D}$, Natan Katz ${ }^{3} \mathbb{D}$, Rafael Gustavo Dal Moro ${ }^{1} \mathbb{D}$, Luiz Felipe Telles ${ }^{5} \mathbb{D}$, Carlos André Aita Schmitz 1 [D

${ }^{1}$ Universidade Federal do Rio Grande do Sul (UFRGS). Porto Alegre, RS, Brasil.

${ }^{2}$ Hospital de Clínicas de Porto Alegre e Universidade Federal do Rio Grande do Sul (UFRGS). Porto Alegre, RS, Brasil.

${ }^{3}$ TelessaúdeRS-UFRGS. Porto Alegre, RS, Brasil.

${ }^{4}$ Universidade Federal de Pelotas (UFPEL). Pelotas, RS, Brasil.

${ }^{5}$ Universidade Luterana do Brasil (ULBRA). Canoas, RS, Brasil.

\section{Resumo}

Novos desafios epidemiológicos e demográficos demandam novas formas de organizar os sistemas de saúde. O presente ensaio propõe a telessaúde como ferramenta organizativa, capaz de suavizar o triângulo de ferro da atenção à saúde e de facilitar a busca pelo triple aim, pelo seu potencial de aumento do acesso e qualidade com redução de custo. A integração da telessaúde ao processo de referência e transição entre serviços assistenciais aumenta a resolutividade da Atenção Primária à Saúde (APS), favorece a coordenação do cuidado, promove adesão terapêutica, diminui reinternações e estimula a prevenção quaternária. Este ensaio propõe a telessaúde como metasserviço que confere densidade tecnológica à APS e permite que ela se torne coordenadora efetiva do cuidado, passando a organizar o fluxo de informações, pessoas e insumos. Frente às inovações propostas, é essencial avaliar o impacto de ações já existentes de telessaúde para viabilizar a sua aplicação como metasserviço de saúde.

Palavras-chave: Telessaúde; Sistemas de Saúde; Inovação

Como citar: Harzheim E, Chueiri PS, Umpierre RN, Gonçalves MR, Siqueira ACS, D’Avila OP, et al. Telessaúde como eixo organizacional dos sistemas universais de saúde do século XXI. Rev Bras Med Fam Comunidade. 2019;14(41):1881. http://dx.doi.org/10.5712/rbmfc14(41)1881

\section{Autor correspondente:}

Erno Harzheim.

E-mail: eharzheim@hcpa.edu.br

Fonte de financiamento: declaram não haver.

Parecer CEP:

não se aplica.

Procedência e revisão por pares: revisado por pares.

Recebido em: 17/09/2018.

Aprovado em: 13/12/2018. 


\begin{abstract}
New epidemiological and demographic challenges require new ways of organizing healthcare systems. This essay proposes telehealth as an organizational tool, capable of smoothing the iron triangle of health care and facilitating achievement of the triple aim, namely greater access and quality with cost reduction. The integration of telehealth into the referral process and the transition between care services increases the resolvability of primary health care (PHC), facilitates the coordination of care, promotes treatment adherence, decreases readmissions, and stimulates quaternary prevention. This essay introduces the notion of telehealth as a meta-service that confers technological density to PHC and allows it to become an effective coordinator of care, organizing the flow of information, people and supplies. In view of the innovations proposed, it is essential to evaluate the impact of existing telehealth initiatives so as to extend their applicability as meta-service.
\end{abstract}

Keywords: Telehealth; Health Systems; Innovation

\title{
Resumen
}

Nuevos desafíos epidemiológicos y demográficos demandan nuevas formas de organizar los sistemas de salud. El presente ensayo propone la telesalud como herramienta organizacional, capaz de suavizar el triángulo de hierro de la atención a la salud y de facilitar la búsqueda por el triple aim, por su potencial de aumento del acceso y calidad con reducción de costo. La integración de la telesalud al proceso de referencia y transición entre servicios asistenciales aumenta la capacidad resolutiva de la Atención Primaria a la Salud (APS), favorece la coordinación del cuidado, promueve la adhesión terapéutica, disminuye reinternaciones y estimula la prevención cuaternaria. Este ensayo propone la telesalud como metaservicio que confiere densidad tecnológica a la APS y permite que ella se convierta en coordinadora efectiva del cuidado, pasando a organizar el flujo de informaciones, personas e insumos. Frente a las innovaciones propuestas, es esencial evaluar el impacto de acciones ya existentes de telesalud para viabilizar su aplicación como metaservicio de salud.

Palabras clave: Telesalud; Sistemas de Salud; Innovación

\section{Introdução}

A produção cientifica de Barbara Starfield confirmou, a partir de estudos comparativos entre sistemas de saúde, que a atenção primária à saúde (APS) deve ser a espinha dorsal dos sistemas nacionais de saúde. No atual momento histórico, mesmo em países ou subsistemas de saúde, públicos ou privados, que tradicionalmente não privilegiaram a APS,${ }^{1}$ grande atenção tem sido dada ao seu poder resolutivo para novos e antigos desafios: dificuldade de acesso, falta de integração das ações assistenciais e, consequentemente, ausência de coordenação do cuidado e elevado custo dos serviços de saúde. ${ }^{2-6}$ Existem evidências de que os sistemas baseados na APS têm melhores resultados sanitários e financeiros, desde que inseridos numa rede organizada de pontos assistenciais. ${ }^{7}$

Para além da capacidade da APS atuar como espinha dorsal nos sistemas de saúde, determinadas mudanças no cenário social, econômico e sanitário impõem a necessidade de repensar a estruturação dos sistemas de saúde. Entre essas mudanças, destacam-se o envelhecimento populacional e o aumento da prevalência de doenças crônicas - um conjunto de demandas cujo advento exige a incorporação de novas tecnologias e medicamentos e o desenvolvimento de diversos tipos de serviços de saúde, como atenção domiciliar, cuidados paliativos, consultas a distância, apoio ao autocuidado/monitoramento e, ainda, outras formas de cuidado continuado., 
Nesse sentido, muitos autores têm direcionado suas pesquisas para uma avaliação mais sistêmica do conjunto de ações e serviços de saúde e das formas de gestão das redes e dos sistemas de saúde. Em 2011, a Organização Pan-Americana da Saúde (OPAS) ${ }^{10}$ propôs os elementos que deveriam constituir redes de atenção à saúde (RAS): população, APS, serviços especializados, sistemas de apoio (diagnóstico, terapêutico, assistência farmacêutica e sistemas de informação), sistemas logísticos (cartão de identificação do usuário, prontuários compartilhados, acesso regulado e o transporte sanitário) e sistema de governança.

Na conceituação das RAS, a telessaúde aparece como sistema de apoio capaz de produzir ajustamento entre pares e de racionalizar a demanda por meio da melhoria do acesso. ${ }^{11}$ No nosso ponto de vista, a telessaúde é muito mais que um sistema de apoio. Com o fortalecimento exponencial das tecnologias de informação e comunicação, a telessaúde deve ocupar o papel de eixo organizador dos sistemas de saúde.

Considerando que a telessaúde apresenta potencial para reformar, transformar e organizar o sistema de saúde ${ }^{12}$ com redução dos custos e ganhos na qualidade, ampliação do acesso e aumento na satisfação do usuário, ${ }^{13,14}$ o presente ensaio apresenta um modelo no qual a telessaúde atua como metasserviço, fortalecendo a APS por meio da oferta de maior densidade tecnológica, estendendo o seu alcance a todos os pontos do sistema e possibilitando a atuação da APS como coordenadora efetiva do sistema de saúde.

\section{Experiências em telessaúde}

O século XXI é caracterizado por avanços exponenciais nas áreas de tecnologia da informação e da comunicação. Nessa perspectiva, a telessaúde é a oferta de serviços de saúde mediada por tecnologia. Pode ser estrategicamente vista como uma inovação disruptiva que coloca em xeque as práticas tradicionais de saúde, com redução de custos e ganhos na qualidade, no acesso e na satisfação do usuário. ${ }^{12-14}$ Algumas revisões sistemáticas já apontam a telessaúde como um fator de suavização do triângulo de ferro da saúde (custo - acesso - qualidade). ${ }^{13-17}$ Portanto, essa estratégia também apoia a busca pelos três objetivos dos sistemas de saúde (triple aim): maior satisfação do usuário, maior qualidade do cuidado e menor custo. ${ }^{18}$

A queda do custo se dá em função da economia de escala; a qualidade resulta de serviços bem estruturados, com profissionais de elevado desempenho clínico e comunicacional, cientificamente embasados, que centralizam e uniformizam a produção do cuidado; e a satisfação do usuário resulta não apenas da qualidade da atenção, mas de sua descentralização. Um exemplo já estabelecido no Brasil, ${ }^{19} \mathrm{e}$ que encontra respaldo nas evidências internacionais, ${ }^{13,14}$ é o do suporte assistencial a distância oferecido a profissionais de saúde: teleconsultorias que ampliam a resolutividade clínica na APS ao mesmo tempo em que diminuem a sobremedicalização e a iatrogenia. ${ }^{19-21}$

Além disso, em diversos lugares do mundo, diferentemente do Brasil, a teleconsulta, entre paciente e profissional de saúde, já é regulamentada e pode ser utilizada na forma de assistência direta a pacientes, sempre integrada aos serviços disponíveis na rede, fortalecendo o papel da APS como ordenadora do cuidado. ${ }^{22,23}$ Apesar de resultados consistentes, há caminho a percorrer para que a telessaúde possa exercer todo seu potencial organizador.

Como toda inovação disruptiva, a telessaúde recebe resistência. Apesar de pouco se saber sobre o perfil de usuários e de não usuários, pesquisas mostram uma subutilização de serviços de telessaúde por médicos da APS (apenas 15\% nos EUA e 10\% no Brasil), ${ }^{24,25}$ sendo uma importante barreira descrita 
a "aceitação clínica", isto é, a disposição de médicos e outros profissionais de saúde em contar com ferramentas de telessaúde dentro de sua rotina diária. ${ }^{26} \mathrm{~A}$ adesão ao uso de tecnologia depende da percepção, por parte do profissional, dos benefícios na sua incorporação.

Tais benefícios podem ser relacionados à facilidade de uso, ganho de tempo, acesso a outros profissionais e novas informações e acesso maior a pacientes, entre outros. Portanto, são necessárias ações não apenas multifacetadas, mas também personalizadas para se lograr êxito na adesão a novas tecnologias. ${ }^{27-29}$ Uma forma de vencer a natural resistência em relação às inovações é a inclusão das ferramentas de telessaúde (exemplo: teleconsulta, teleconsultoria, telediagnóstico e telemonitoramento) como mecanismos de coordenação assistencial, ${ }^{21}$ de regulação do acesso (sistema de referência e contrarreferência) $)^{20,30}$ e de transição de cuidado (APS-Hospital-Domicílio). ${ }^{31,32}$

Outra forma de vencer as barreiras iniciais é permitir que a telessaúde também seja ofertada por meio de interação direta com os usuários do sistema de saúde, ${ }^{22,23}$ já que amplia diretamente o acesso. Inclusive, há mais aceitação pelo uso da telessaúde entre usuários (pacientes) do que entre provedores de saúde. ${ }^{13}$ Os usuários dos sistemas de saúde estão, na maior parte das vezes, livres de pressões corporativas, e optam autonomamente por opções de cuidado que lhes parecem mais acessíveis e efetivas.

Entretanto, enquanto a telessaúde for vista e utilizada apenas como serviço, o seu potencial de transformação não poderá ser totalmente explorado. Nesse sentido, são os processos de gestão e coordenação assistencial que fazem crescer de forma intensa e rápida a influência da telessaúde:12 por exemplo, ao ser incorporada ao processo de referência da APS para a atenção especializada ou no momento de transição de cuidado do hospital para a APS ou, ainda, na transição do cuidado ambulatorial especializado para a APS. Dessa forma, a telessaúde aumenta a resolutividade, facilita a coordenação do cuidado e a adesão terapêutica, inibe as reinternações e estimula a prática da prevenção quaternária. . $3,14,19,31,32^{\text {Mas, } 0}$ papel da telessaúde pode ser ainda maior: a telessaúde pode ser o tecido de integração das RAS.

\section{Uma nova proposta: telessaúde como metasserviço de saúde}

A partir do contexto tecnológico do século $\mathrm{XXI}$ e das diretrizes sobre as RAS preconizadas pela OPAS, ${ }^{10}$ os autores deste ensaio propõem uma nova sistematização acerca da organização dos sistemas de saúde, exemplificada na Figura 1.

No centro dessa sistematização estão as pessoas, ${ }^{12,33}$ que devem ter amplo acesso a serviços de Atenção Primária formados por equipes multiprofissionais, com subsidiariedade, resolutividade e grande incorporação tecnológica. É essencial, porém, que a centralização nas pessoas se consolide não apenas nos processos assistenciais, mas também nas ações logísticas e de apoio, que incluem os sistemas de informação, os registros eletrônicos em saúde, a assistência farmacêutica, o apoio diagnóstico e terapêutico, o transporte e a regulação clínica.

Considerando a conformação do Estado brasileiro e do nosso do sistema de saúde, seria essencial que os sistemas de informação e os registros eletrônicos interoperassem de acordo com uma parametrização federal, garantindo assim um fluxo de informação corrente (livre) entre diferentes entes federados, seus serviços de saúde e, inclusive, transpondo a segmentação peculiar à nossa esquizofrenizante separação entre sistema público e privado, ainda vigente em um contexto de sistema único de saúde. 


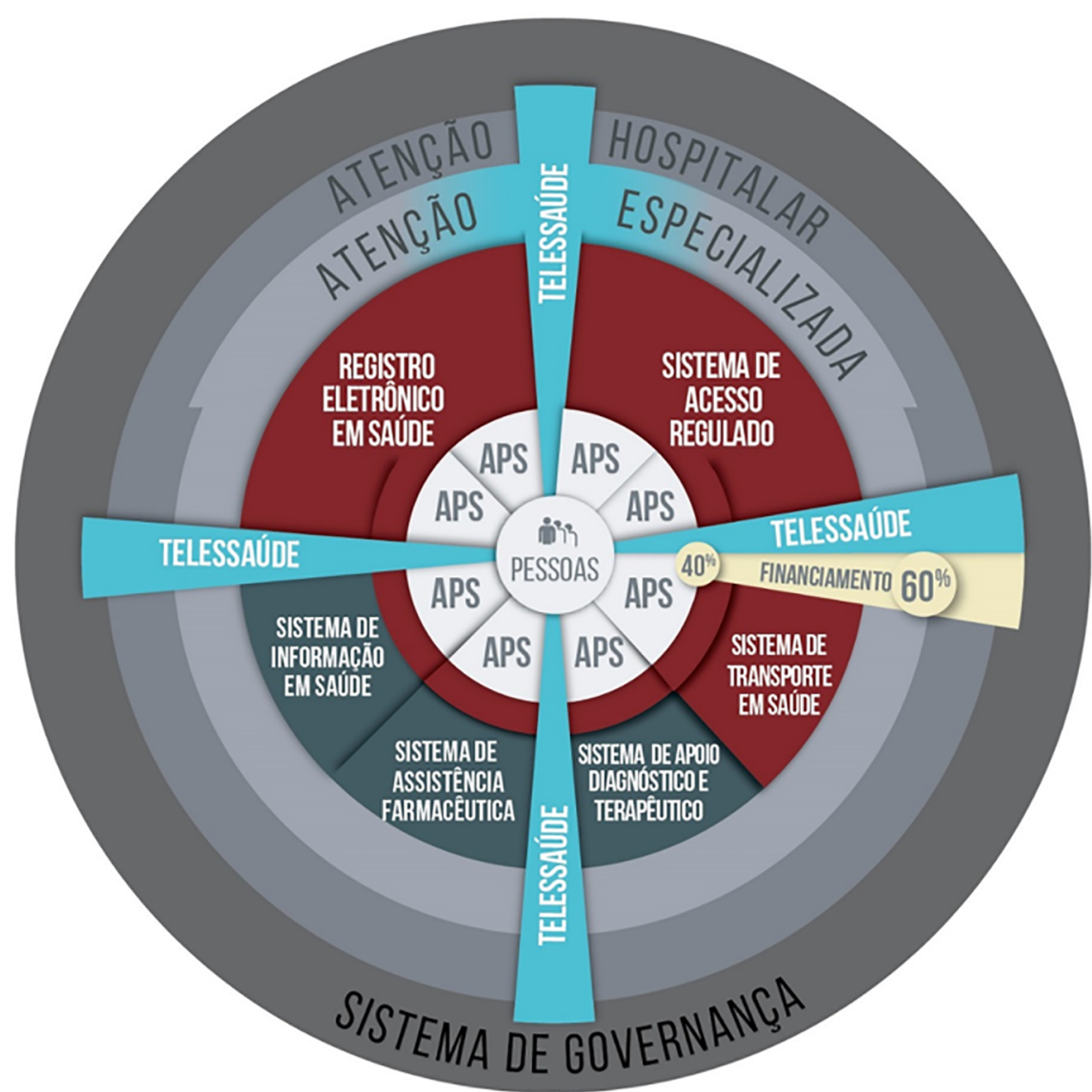

Sistema logístico

Sistema de apoio

Figura 1. Telessaúde como meta serviço de saúde. Telessaúde RS-UFRGS, Porto Alegre, 2017. APS=Atenção Primária à Saúde. $40 \%=$ até $40 \%$ de financiamento para APS. $60 \%=$ financiamento da atenção hospitalar, especializada e outros setores que não APS.

O círculo mais externo representa a governança do sistema, que deve dar coesão à rede de ações e serviços, trabalhando a partir dos princípios de transparência, accountability, participação dos indivíduos que compõem a RAS e também garantir coerência entre as ações e os objetivos almejados (triple aim). ${ }^{10,18}$

O modelo proposto também incorpora os percentuais do orçamento da saúde que devem ser destinados aos serviços de saúde de atenção primária e especializada baseados na informação que os sistemas de saúde de países com APS forte, ou mesmo aqueles onde a APS está em expressivo desenvolvimento, buscam investir entre $20 \%$ e $40 \%$ do orçamento total de saúde para financiamento de cuidados primários. ${ }^{34,35}$

A Figura 1 mostra que, quando as necessidades em saúde não são atendidas no escopo da APS, devem ser referidas para atenção especializada e/ou hospitalar de forma regulada por meio de critérios clínicos embasados na melhor evidência cientifica disponível e apoiada por processos de telessaúde e pelo sistema de informação. Os serviços especializados assumem assim o papel de complementação e 
de recurso para $\mathrm{APS},{ }^{30}$ enquanto as centrais de regulação-telessaúde surgem como apoio à APS no seu papel cada vez mais difícil de coordenar o cuidado frente à rede (quase) infinita de recursos, muitas vezes redundantes e inefetivos. ${ }^{20,21}$

Com essas características, a telessaúde pode produzir inteligência e efetividade para o sistema de regulação ao dar agilidade ao fluxo de informação, de recursos financeiros e de pessoas pelo sistema. Isso ocorre porque os reguladores na Telessaúde, em contato direto com as equipes de APS e numa via de mão dupla, incorporam também a função de teleconsultores e podem lançar mão de todas as ferramentas de telessaúde para evitar ou qualificar encaminhamentos da APS para outros níveis de atenção. ${ }^{20,21}$

A central de regulação/telessaúde também tem acesso privilegiado aos demais sistemas logísticos e aos sistemas de apoio, facilitando, de acordo com a prioridade clínica, o fluxo dos pacientes entre serviços, direcionando-os para os serviços mais qualificados e acessíveis para resolver cada problema. A vantagem da regulação/telessaúde em refinar este fluxo é a disponibilidade, em um clique, de todas as informações e subsídios necessários: demanda assistencial, oferta de serviços, protocolos sitematizados e evidências robustas atualizadas. O mesmo refinamento ocorre com os conjuntos de dados mínimos para armazenamento e fluxo de informações, bem como com as relações humanas, administrativas e políticas envolvidas nos processos. Tanto maior será esse efeito quanto mais linhas de transição do cuidado forem envolvidas (APS-Hospital-Domicílio).

Nesse novo desenho da rede (Figura 1), a telessaúde deixa de ser vista como um serviço de apoio e passa a ter inserção e função na prática diária. De um lado, a telessaúde ainda exerce o tradicional papel de serviço assistencial que oferta cuidado usual (consultas, exames), embora de forma mediada pela tecnologia (por exemplo: telediagnóstico, teleconsulta, teleconsultoria), com caráter aditivo ou substitutivo a serviços previamente existentes, principalmente em locais sem acesso ou com baixo interesse dos prestadores de atenção especializada.

Por outro, assume um papel disruptivo e inovador, passando a operar também como metasserviço de saúde, permeando as ações de saúde, fortalecendo a união entre os pontos assistenciais e regulando o acesso aos serviços especializados. Metasserviço, pois, valendo-se da virtualização da distância, da capilaridade e do ajustamento entre pares, terá função de integrar a tomada de decisão clínica e gerencial e os fluxos de informações, servindo como serviço de saúde dos serviços de saúde.

A partir desse cenário de dupla inserção (serviço e metasserviço), a telessaúde pode tornar-se a organizadora do fluxo de pessoas, de informações e de insumos, inclusive financeiros, entre os pontos de atenção. Será da telessaúde o papel de ordenação da APS, qualificando o deslocamento físico de pacientes - pacientes certos, no lugar certo e na hora certa - ${ }^{10,21}$ com redução de custos e riscos, garantindo o uso das melhores práticas assistenciais, ampliando a qualidade e aumentando a resolutividade da APS, expandindo assim a integralidade do cuidado (carteira de serviços). ${ }^{13,14}$ Nessa proposta, a telessaúde modelará e participará da regulação dos caminhos virtuais e físicos percorridos por pessoas e informações, tornando-se estratégica, para a governança e o financiamento, como eixo organizacional de alta capilaridade que busca ampliar o acesso, garantir a qualidade e otimizar recursos. 
Ao formar uma rede capilarizada de comunicação para replicar informações consistentes e seguras de forma ágil e multidirecional entre grupos geograficamente dispersos, a telessaúde é capaz de aliar, de forma híbrida, novas tecnologias (internet, dispositivos móveis, sensores) e velhas tecnologias (rádio, telefonia convencional) em ações multifacetadas e personalizadas. Isso é especialmente válido para sistemas de saúde e de informação com baixa interoperabilidade, o que inclui a maioria dos países de baixa/média renda e vários de alta renda. ${ }^{36}$

\section{Conclusões}

Sabe-se que a busca por inovações pode contribuir para a construção e a gestão de sistemas de saúde mais adequados ao atual contexto. ${ }^{37}$ As novas tecnologias, sem as amarras regulamentadoras e a tendência de planificação estatal, mas fortemente embasadas na melhor evidência científica disponível, podem ser as ferramentas para o enfrentamento dos desafios destacados acima. Considerando que os problemas de qualidade e os resultados sanitários e econômicos dos sistemas de saúde estão mais relacionados a limitações do sistema em si do que a erros ou ações individuais, ${ }^{38}$ há forte necessidade de repensar a forma de organização dos sistemas. Essa nova forma deve incluir os aspectos já consolidados, como uma forte base na APS, ${ }^{39}$ assim como dar os saltos de evolução necessários para enfrentar os novos desafios que a saúde do século XXI nos impõe, livres de regulamentação corporativa e repressora a inovações.

A incorporação maciça de tecnologias de informação e comunicação nos moldes da telessaúde aqui apresentados pode representar o salto de evolução organizativa que garanta sobrevida aos sistemas universais de saúde. Evidentemente, deve haver cautela na adoção indiscriminada da telessaúde, havendo necessidade contínua de estudos sobre sua efetividade e custo-efetividade. ${ }^{40-43}$ Fica claro que o próximo passo, após este ensaio, é avaliar os resultados de experiência de telessaúde ora em andamento, considerando, a partir disso, a implementação da telessaúde como metasserviço.

\section{Contribuição dos autores}

Erno Harzheim - responsável pela concepção da ideia e pela redação final do artigo;

Carlos André Aita Schmitz - responsável pela pesquisa bibliográfica, organização do trabalho, redação final do artigo;

Patrícia Chueiri, Cynthia Molina Bastos, Natan Katz, Otávio D’Avila - contribuíram na pesquisa bibliográfica e na redação do artigo;

Roberto Nunes Umpierre, Marcelo Rodrigues Gonçalves, Ana Célia da Silva Siqueira, Rafael Gustavo dal Moro, Luiz Felipe Telles - contribuíram com sugestões para o referencial teórico e participaram das revisões do artigo.

Todos os autores aprovaram a versão final e concordaram com prestar contas sobre todos os aspectos do trabalho. 


\title{
Conflito de interesses
}

\author{
Declaram não haver.
}

\section{Referências}

1. Koller CF, Khullar D. Primary care spending rate - A lever for encouraging investment in primary care. N Engl J Med. 2017;377(18):170911. http://dx.doi.org/10.1056/NEJMp1709538

2. Bodenheimer T. The future of primary care: transforming practice. N Engl J Med. 2008;359(20):2086-9. http://dx.doi.org/10.1056/ NEJMp0805631

3. Gauthier AK, Davis K, Schoenbaum SC. Commentary--Achieving a high-performance health system: High reliability organizations within a broader agenda. Health Serv Res. 2006;41(4 Pt 2):1710-20.

4. Hofmarcher MM, Oxley H, Rusticelli E. Improved health system performance through better care coordination. Paris: OECD Health Working Papers; 2007.

5. Schoen C, Osborn R, Squires D, Doty M, Pierson R, Applebaum S. New 2011 survey of patients with complex care needs in eleven countries finds that care is often poorly coordinated. Health Aff (Millwood). 2011;30(12):2437-48. http://dx.doi.org/10.1377/hlthaff.2011.0923

6. Wakeman SE, Barnett ML. Primary Care and the Opioid-Overdose Crisis - Buprenorphine Myths and Realities. N Engl J Med. 2018;379(1):14. http://dx.doi.org/10.1056/NEJMp1802741

7. Starfield B, Shi L. Policy relevant determinants of health: an international perspective. Health Policy. 2002;60(3):201-18. http://dx.doi. org/10.1016/S0168-8510(01)00208-1

8. Thorpe KE, Howard DH. The rise in spending among Medicare beneficiaries: the role of chronic disease prevalence and changes in treatment intensity. Health Aff (Millwood). 2006;25(5):w378-88.

9. Chueiri PS, Harzheim E, Gauche H, Vasconcelos LLC. Pessoas com doenças crônicas, as redes de atenção e a Atenção Primária à Saúde. Divulg Saúde Debate. 2014;52:114-24.

10. Mendes EV. As redes de atenção à saúde. Brasília: Organização Pan-Americana da Saúde; 2011.

11. Mendes EV. A construção social da atenção primária à saúde. Brasília: Conselho Nacional de Secretários de Saúde; 2015.

12. Schwamm LH. Telehealth: seven strategies to successfully implement disruptive technology and transform health care. Health Aff (Millwood). 2014;33(2):200-6. http://dx.doi.org/10.1377/hlthaff.2013.1021

13. Bashshur RL, Howell JD, Krupinski EA, Harms KM, Bashshur N, Doarn CR. The Empirical Foundations of Telemedicine Interventions in Primary Care. Telemed J E Health. 2016;22(5):342-75. http://dx.doi.org/10.1089/tmj.2016.0045

14. Bashshur RL, Shannon GW, Smith BR, Alverson DC, Antoniotti N, Barsan WG, et al. The empirical foundations of telemedicine interventions for chronic disease management. Telemed J E Health. 2014;20(9):769-800. http://dx.doi.org/10.1089/tmj.2014.9981

15. Dixon J, Welch HG. Priority setting:lessons from Oregon. Lancet. 1991;337(8746):891-4. http://dx.doi.org/10.1016/0140-6736(91)90213-9

16. Kissick W. Medicine's dilemmas: Infinite needs versus finite resources. New Haven:Yale University Press; 1994.

17. Sabik LM, Lie RK. Priority setting in health care: Lessons from the experiences of eight countries. Int J Equity Health. 2008;7:4. http:// dx.doi.org/10.1186/1475-9276-7-4

18. Berwick DM, Nolan TW, Whittington J. The triple aim: care, health, and cost. Health Aff (Millwood). 2008;27(3):759-69. http://dx.doi. org/10.1377/hlthaff.27.3.759

19. Harzheim E, Goncalves MR, Umpierre RN, da Silva Siqueira AC, Katz N, Agostinho MR, et al. Telehealth in Rio Grande do Sul, Brazil: Bridging the gaps. Telemed J E Health. 2016;22(11):938-44. http://dx.doi.org/10.1089/tmj.2015.0210

20. Schmitz CAA, Siqueira ACS, Gonçalves MR, Castro Filho ED, Harzheim E. Telessaúde na Atenção Primária à Saúde. In: Gusso G, Lopes JMC, Dias LC, eds. Tratado de Medicina de Família e Comunidade: Princípios, Formação e Prática. 2ª ed. Porto Alegre: Artmed; 2019. p. 165-76.

21. Harzheim E, Siqueira ACS, Katz N, Dal Moro RG, Bastos CGM, D’Avila OP, et al. Telemedicina como motor da coordenação assistencial: muito além da tecnologia. In: Barbosa AF, ed. Pesquisa sobre o uso das Tecnologias de Informação e Comunicação nos estabelecimentos de saúde brasileiros: TIC Saúde 2015. São Paulo: Comitê Gestor da Internet no Brasil; 2016. p. 93-102. 
22. Schmitz CAA, Gonçalves MR, Umpierre RN, Siqueira ACS, D’Ávila OP, Bastos CGM, et al. Teleconsulta: nova fronteira da interação entre médicos e pacientes. Rev Bras Med Fam Comunidade. 2017;12(39):1-7. http://dx.doi.org/10.5712/rbmfc12(39)1540

23. Molina-Bastos CG, D’Avila OP, Schmitz CAA. Atendimento em saúde por meio de recursos digitais. In: Gusso G, Lopes JMC, Dias LC, eds. Tratado de Medicina de Família e Comunidade: Princípios, Formação e Prática. 2ª ed. Porto Alegre: Artmed; 2019. p. $159-64$.

24. Moore MA, Coffman M, Jetty A, Klink K, Petterson S, Bazemore A. Family Physicians Report Considerable Interest in, but Limited Use of, Telehealth Services. J Am Board Fam Med. 2017;30(3):320-30. http://dx.doi.org/10.3122/jabfm.2017.03.160201

25. Schmitz CAA, Harzheim E. Oferta e utilização de teleconsultorias para Atenção Primária à Saúde no Programa Telessaúde Brasil Redes. Rev Bras Med Fam Comunidade. 2017;12(39):1-11.

26. Wade VA, Eliott JA, Hiller JE. Clinician acceptance is the key factor for sustainable telehealth services. Qual Health Res. 2014;24(5):68294. http://dx.doi.org/10.1177/1049732314528809

27. Oxman AD, Thomson MA, Davis DA, Haynes RB. No magic bullets: a systematic review of 102 trials of interventions to improve professional practice. CMAJ. 1995;153(10):1423-31.

28. Wong G, Greenhalgh T, Pawson R. Internet-based medical education: a realist review of what works, for whom and in what circumstances. BMC Med Educ. 2010;10:12. http://dx.doi.org/10.1186/1472-6920-10-12

29. Davis DA, Thomson MA, Oxman AD, Haynes RB. Changing physician performance. A systematic review of the effect of continuing medical education strategies. JAMA. 1995;274(9):700-5. http://dx.doi.org/10.1001/jama.1995.03530090032018

30. Liddy C, Rowan MS, Afkham A, Maranger J, Keely E. Building access to specialist care through e-consultation. Open Med. 2013;7(1):e1-8.

31. Moreira AM, Marobin R, Rados DV, de Farias CB, Coelli S, Bernardi BL, et al. Effects of nurse telesupport on transition between specialized and primary care in diabetic patients: study protocol for a randomized controlled trial. Trials. 2017;18(1):222. http://dx.doi.org/10.1186/ s13063-017-1954-z

32. Cawthon C, Walia S, Osborn CY, Niesner KJ, Schnipper JL, Kripalani S. Improving care transitions: the patient perspective. J Health Commun. 2012;17 Suppl 3:312-24.

33. Gérvas J, Pané Mena O, Sicras Mainar A; Seminario Innovación en Atención Primaria. The response capacity of primary care and the service networks. The case of Catalonia's Hospital Consortium. Aten Primaria. 2006;38(9):506-10.

34. Kringos DS, Boerma WGW, Hutchinson A, Saltman RB, eds. Building primary care in a changing Europe. London: The European Observatory on Health Systems and Policies; 2015.

35. Mendes A, Marques RM. O financiamento da Atenção Básica e da Estratégia Saúde da Família no Sistema Único de Saúde. Saúde Debate. 2014;38(103):900-16.

36. Fragidis LL, Chatzoglou PD. Implementation of a nationwide electronic health record (EHR). Int J Health Care Qual Assur. 2018;31(2):11630. http://dx.doi.org/10.1108/IJHCQA-09-2016-0136

37. Lyngsø AM, Godtfredsen NS, Høst D, Frølich A. Instruments to assess integrated care: a systematic review. Int J Integr Care.2014; 14:e027.

38. Couto RC, Pedrosa TMG, Rosa MB. Erros acontecem: a força da transparência para o enfrentamento dos eventos adversos assistenciais em pacientes hospitalizados. Construindo um sistema de saúde mais seguro. Belo Horizonte: Instituto de Estudos de Saúde Suplementar; 2016.

39. Gillies RR, Chenok KE, Shortell SM, Pawlson G, Wimbush JJ. The impact of health plan delivery system organization on clinical quality and patient satisfaction. Health Serv Res. 2006;41(4 Pt 1):1181-99.

40. Ekeland AG, Bowes A, Flottorp S. Effectiveness of telemedicine: a systematic review of reviews. Int J Med Inform. 2010;79(11):736-71. http://dx.doi.org/10.1016/j.ijmedinf.2010.08.006

41. Lustig TA. The role of telehealth in an evolving health care environment: workshop summary. Washington: National Academies Press; 2012.

42. Wootton R. Twenty years of telemedicine in chronic disease management--an evidence synthesis. J Telemed Telecare. 2012;18(4):21120. http://dx.doi.org/10.1258/jtt.2012.120219

43. Tuckson RV, Edmunds M, Hodgkins ML. Telehealth. N Engl J Med. 2017;377(16):1585-92. http://dx.doi.org/10.1056/NEJMsr1503323 